\title{
Fractal Trap for The Chaotic Behavior Of Real Cubic Polynomials
}

\author{
${ }^{1}$ R. S. Thakkar, ${ }^{2}$ P.J.Bhatt \\ ${ }^{I}$ Department of Mathematics, Tolani College of Science, Adipur. Gujarat. India \\ ${ }^{2}$ Nar Narayan Shastri Institute of Technology, Ahmedabad. Gujarat. India
}

\begin{abstract}
A real quadratic family functions $f(x)=k x(1-x), k \in R$, has indicated that even the simplest looking functions may have the complicated dynamics. This logistic map exhibits the properties like topological transitivity, sensitivity dependence on initial conditions and density of periodic points. The cubic family functions $f: R \rightarrow R, f(x)=x^{3}+\lambda x, \lambda \in R$, are no exceptions. The dynamics of this family is 'controlled' for an interval of values of $\lambda$, but it becomes more complicated as the value of $\lambda$ decreases from -1.5. At $\lambda=-3, f(x)$ is chaotic on the interval $[-2,2]$. In this paper, we shall see that the chaotic behaviour of $f(x)=x^{3}+\lambda x$ is even more complex for $\lambda<-3$.
\end{abstract}

Key words: Dense periodic points, Domain of chaos, Dynamics of a function, Fractal, Sensitivity dependence on initial conditions

\section{Introduction}

The realization of significance of nonlinearity in Mathematical applications gave rise to a new field of Mathematics, which is now popularly known as : Chaos Theory and Dynamical Systems. In fact, this field has been considered as one of the most significant breakthroughs in Mathematics in the last century. In particular, its applications in a wide range of subjects including Physics, Biology, Chemistry, Ecology, Fluid Mechanics, Engineering, Economics etc., have made the field very attractive and important for the researchers from various disciplines.[1] The most exciting fact in the field of Dynamical Systems is that some simplest looking nonlinear maps do exhibit very complicated behaviour. In fact, some such maps illustrate virtually every important phenomenon that occur in the Dynamical Systems. [2] A real quadratic family [3] $f(x)=k x(1-x), k \in R$ (set of real numbers), has resolutely indicated that even the simplest looking functions may have the complex dynamics. This indication has put every real function under suspicion and the simple looking cubic family functions of the form $\mathrm{f}: \mathrm{R} \rightarrow \mathrm{R}, \mathrm{f}(\mathrm{x})=\mathrm{x}^{3}+\lambda \mathrm{x}, \lambda \in \mathrm{R}$, are no exceptions. It is easy to see that these cubic functions have simple dynamics for $-1.5 \leq \lambda<\infty$. However, the dynamics of this family becomes more and more complicated as $\lambda$ decreases from -1.5 . At $\lambda=-3$, $\mathrm{f}(\mathrm{x})$ exhibits topological transitivity along with dense periodic points and hence turns out to be chaotic on $[-2,2]$. In this paper, we shall see that the chaotic behaviour of $f(x)=x^{3}+\lambda x$ is even more complex for $\lambda<-3$ than that is at $\lambda=-3$.

We mention few elementary results about $f(x)=x^{3}+\lambda x$ (proofs for these results are simple and hence omitted)

Lemma 1.1 : Let $f(x)=x^{3}+\lambda x, \lambda \in R$. Then,

a)

For $\lambda>1, \mathrm{x}=0$ is a repelling fixed point of $\mathrm{f}(\mathrm{x})$ and for $\lambda=1, \mathrm{x}=0$ is a non-hyperbolic fixed

point.

b) For $\lambda<1$,

(1) $\mathrm{P}_{\lambda}$ and $-\mathrm{P}_{\lambda}$ are repelling fixed points where $\mathrm{P}_{\lambda}=\sqrt{1-\lambda}$.

( 2 ) 0 is an attracting fixed point if $-1<\lambda<1$, but it is a repelling fixed point for $\lambda<-1$. For $\lambda=$ $-1,0$ is a non-hyperbolic fixed point of $\mathrm{f}(\mathrm{x})$.

Lemma 1.2 : Let $f(x)=x^{3}+\lambda x$ where $\lambda<1$. Then if $x>P_{\lambda}$, then $f^{n}(x) \rightarrow \infty$ as $n \rightarrow \infty$. Similarly, if $\mathrm{x}<-\mathrm{P}_{\lambda}$, then $\mathrm{f}^{\mathrm{n}}(\mathrm{x}) \rightarrow-\infty$ as $\mathrm{n} \rightarrow \infty$. 
This lemma suggests that the points of $\mathrm{R}-\left(-\mathrm{P}_{\lambda}, \mathrm{P}_{\lambda}\right)$ are approaching to $\pm \infty$ under the iterations of $\mathrm{f}$. We also observe that the local extreme values of $\mathrm{f}$ are given by $\pm \mathrm{f}(\sqrt{-\lambda / 3})$. Also, as $\lambda$ decreases from $0, \mid \mathrm{f}($ $\sqrt{-\lambda / 3}) \mid$ increases. For $-3 \leq \lambda<0,|\mathrm{f}(\sqrt{-\lambda / 3})|$ lies in $\left[-\mathrm{P}_{\lambda}, \mathrm{P}_{\lambda}\right]$ and at $\lambda=-3, \quad|\mathrm{f}(\sqrt{-\lambda / 3})|=$ $\mathrm{P}_{\lambda}$. But for $\lambda<-3,|\mathrm{f}(\sqrt{-\lambda / 3})|>\mathrm{P}_{\lambda}$ and hence doesn't lie in $\left[-\mathrm{P}_{\lambda}, \mathrm{P}_{\lambda}\right]$.

This simple fact leads towards a complex dynamics of $\mathrm{f}$ for $\lambda<-3$. At every iteration of $\mathrm{f}$, there are some subintervals of $\left[-\mathrm{P}_{\lambda}, \mathrm{P}_{\lambda}\right]$, which are mapped outside $\left[-\mathrm{P}_{\lambda}, \mathrm{P}_{\lambda}\right]$. So the points of these subintervals move towards $\pm \infty$ under the iterations of $f$, and dynamics of $f$ on these intervals is simple. But the set of remaining points of $\left[-\mathrm{P}_{\lambda}, \mathrm{P}_{\lambda}\right]$ ( which are never mapped outside of $\left[-\mathrm{P}_{\lambda}, \mathrm{P}_{\lambda}\right]$ under the iterations of $\mathrm{f}$ ) is very interesting and the behaviour of $\mathrm{f}$ on this set is discussed now. First we find out the subintervals of $\left[-\mathrm{P}_{\lambda}, \mathrm{P}_{\lambda}\right]$, which are mapped out of $\left[-P_{\lambda}, P_{\lambda}\right]$ under the first iteration of $f(x)=x^{3}+\lambda x$ for $\lambda<-3$.

Lemma 1.3: Let $f:\left[-P_{\lambda}, P_{\lambda}\right]$ T R, $f(x)=x^{3}+\lambda x, \lambda<-3$. Then

a) There exists numbers $r$ and s with $r \in(0, \sqrt{-\lambda / 3}), s \in(\sqrt{-\lambda / 3}, \sqrt{-\lambda})$ and $f(r)=f(s)=-P_{\lambda}$. For any $\mathrm{t} \in(\mathrm{r}, \mathrm{s}), \mathrm{f}(\mathrm{t})<-\mathrm{P}_{\lambda}$. Hence $\mathrm{f}^{\mathrm{n}}(\mathrm{t}) \rightarrow-\infty$ as $\mathrm{n} \rightarrow \infty$.

b) $\quad 11$ points of $\left[0, P_{\lambda}\right]-(r, s)$ remain in $\left[-P_{\lambda}, P_{\lambda}\right]$ under $f$. Proof is simple and hence omitted.

- $\quad$ Since $\mathrm{f}$ is an odd function, similar arguments will give the next result as follows :

Lemma 1.4: Let $f:\left[-P_{\lambda}, P_{\lambda}\right] \rightarrow R, f(x)=x^{3}+\lambda x, \lambda<-3$. Then for the values of $r$ and $s$ mentioned in lemma 1 ,

a) $\quad-\mathrm{r} \in(-\sqrt{-\lambda / 3}, 0),-\mathrm{s} \in(-\sqrt{-\lambda},-\sqrt{-\lambda / 3})$ and $\mathrm{f}(-\mathrm{r})=\mathrm{f}(-\mathrm{s})=\mathrm{P}_{\lambda}$

b) $\quad$ For any $t \in(-s,-r), f(t)>P_{\lambda}$. Hence $f^{n}(t) \rightarrow \infty$ as $n \rightarrow \infty$.

c) All points of $\left[-P_{\lambda}, 0\right]-(-s,-r)$ remain in $\left[-P_{\lambda}, P_{\lambda}\right]$ under $f$.

\section{The Construction Of Domain Of Chaos}

The lemmas 1.3 and 1.4 suggest that $\left[0, \mathrm{P}_{\lambda}\right]$ is divided into four subintervals $[0, \mathrm{r}],(\mathrm{r}, \mathrm{s})$, $[\mathrm{s}, \sqrt{-\lambda}]$ and $\left[\sqrt{-\lambda}, \mathrm{P}_{\lambda}\right]$ when the restriction of $\mathrm{f}$ is applied to it, i.e., by considering $\mathrm{f}_{[0, \mathrm{P} \lambda]}:\left[0, \mathrm{P}_{\lambda}\right] \rightarrow$ $\mathrm{R}$, which is one-one. Out of these four subintervals, $(\mathrm{r}, \mathrm{s})$ is mapped out of $\left[-\mathrm{P}_{\lambda}, \mathrm{P}_{\lambda}\right]$ and travels to $-\infty$ under the iterations of $\mathrm{f}$. The remaining three subintervals are either mapped onto $\left[0, \mathrm{P}_{\lambda}\right]$ or $\left[-\mathrm{P}_{\lambda}, 0\right]$ under the first iteration of $\mathrm{f}$. (See the next figure) The case for $\quad\left[-\mathrm{P}_{\lambda}, 0\right]$ is similar except that the 'escaping interval' travels towards $+\infty$ under the iterations of $\mathrm{f}$. On the whole, after the first iteration of $f$ on $\left[-\mathrm{P}_{\lambda}, \mathrm{P}_{\lambda}\right]$, the interval is divided into eight subintervals with two of the subintervals escaping out of $\quad\left[-\mathrm{P}_{\lambda}, \mathrm{P}_{\lambda}\right]$ ( and traveling to $\pm \infty$ under iterations of $\mathrm{f}$ ) and the remaining six subintervals mapping onto either $\left[0, \mathrm{P}_{\lambda}\right]$ or $\left[-\mathrm{P}_{\lambda}, 0\right]$.

This analysis helps to understand the second iteration of $\mathrm{f}$. Each of the remaining subintervals of $\left[-\mathrm{P}_{\lambda}, \mathrm{P}_{\lambda}\right]$ (i.e. each of $\left[-\mathrm{P}_{\lambda},-\sqrt{-\lambda}\right],[-\sqrt{-\lambda},-\mathrm{s}],[-\mathrm{r}, 0],[0, \mathrm{r}],[\mathrm{s}, \sqrt{-\lambda}]$ and $\left[\sqrt{-\lambda}, \mathrm{P}_{\lambda}\right]$ ), all of which are mapped under one-one correspondence onto $\left[0, \mathrm{P}_{\lambda}\right]$ or $\left[-\mathrm{P}_{\lambda}, 0\right]$. under the second iteration $\mathrm{f}^{2}$, gives rise to four more subintervals of itself, with one of those subintervals escaping from $\left[-\mathrm{P}_{\lambda}, \mathrm{P}_{\lambda}\right]$ and the remaining three getting mapped onto either $\left[0, \mathrm{P}_{\lambda}\right]$ or $\left[-\mathrm{P}_{\lambda}, 0\right]$. For example, take the subinterval $\left[\sqrt{-\lambda}, \mathrm{P}_{\lambda}\right]$, which is mapped onto $\left[0, \mathrm{P}_{\lambda}\right]$ under the one-one correspondence of the restricted $\mathrm{f}$. (Similar things hold for the other five subintervals.) $\left[\sqrt{-\lambda}, \mathrm{P}_{\lambda}\right]$ is divided into the four subintervals as shown in the following figure :

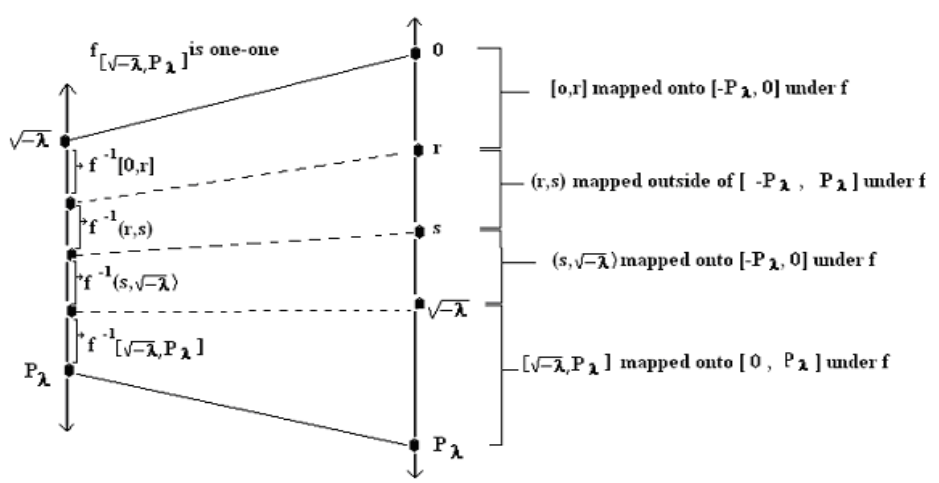

www.iosrjournals.org 
$\mathrm{f}:\left[\sqrt{-\lambda}, \mathrm{P}_{\lambda}\right]$ to $\left[0, \mathrm{P}_{\lambda}\right]$ is one-one. Thus,

$$
\begin{aligned}
\mathrm{f}^{2}\left[\sqrt{-\lambda}, \mathrm{P}_{\lambda}\right] & =\mathrm{f}\left[0, \mathrm{P}_{\lambda}\right] \\
= & \mathrm{f}\left([0, \mathrm{r}] \cup(\mathrm{r}, \mathrm{s}) \cup[\mathrm{s}, \sqrt{-\lambda}] \cup\left[\sqrt{-\lambda}, \mathrm{P}_{\lambda}\right]\right)
\end{aligned}
$$

So, $\left[\sqrt{-\lambda}, \mathrm{P}_{\lambda}\right]=\mathrm{f}^{-1}([0, \mathrm{r}]) \cup \mathrm{f}^{-1}((\mathrm{r}, \mathrm{s})) \cup \mathrm{f}^{-1}([\mathrm{~s}, \sqrt{-\lambda}]) \cup \mathrm{f}^{-1}\left(\left[\sqrt{-\lambda}, \mathrm{P}_{\lambda}\right]\right)$

If $\mathrm{A}=\left[\sqrt{-\lambda}, \mathrm{P}_{\lambda}\right]$ then $\mathrm{f}_{\mathrm{A}}^{-1}([0, \mathrm{r}]), \mathrm{f}_{\mathrm{A}}^{-1}([\mathrm{~s}, \sqrt{-\lambda}])$ and $\mathrm{f}_{\mathrm{A}}^{-1}\left(\left[\sqrt{-\lambda}, \mathrm{P}_{\lambda}\right]\right)$ are the closed intervals and $\mathrm{f}_{\mathrm{A}}{ }^{-1}((\mathrm{r}, \mathrm{s}))$ is an escaping interval, which is mapped out of $\left[-\mathrm{P}_{\lambda}, \mathrm{P}_{\lambda}\right]$. This shows that $\left[\sqrt{-\lambda}, \mathrm{P}_{\lambda}\right]$ can be seen as divided into four subintervals ( if they overlap, they overlap only on boundaries ), out of which one is escaping out of $\left[-\mathrm{P}_{\lambda}, \mathrm{P}_{\lambda}\right]$.

Hence, at the end of the second iteration of $\left[-\mathrm{P}_{\lambda}, \mathrm{P}_{\lambda}\right]$ under $\mathrm{f}$, we see that six more subintervals escape from $\left[-P_{\lambda}, P_{\lambda}\right]$ and eighteen subintervals of $\left[-P_{\lambda}, P_{\lambda}\right]$ are seen to be mapped onto $\left[0, P_{\lambda}\right]$ or $\left[-P_{\lambda}, 0\right]$.

Continuing the process of taking iterations $f, f^{2}, f^{3}, \ldots \ldots$ of the points from $\left[-P_{\lambda}, P_{\lambda}\right]$ to $R$, we see that more and more subintervals keep escaping from $\left[-P_{\lambda}, P_{\lambda}\right]$ and iterating towards $\pm \infty$ under the iterations of $f$. And the number of remaining subintervals in $\left[-\mathrm{P}_{\lambda}, \mathrm{P}_{\lambda}\right]$ increase under the iterations of $\mathrm{f}$, with the length of these remaining intervals getting smaller and smaller. For example, at the end of two iterations, eight subintervals of $\left[-\mathrm{P}_{\lambda}, \mathrm{P}_{\lambda}\right]$ are mapped out of $\left[-\mathrm{P}_{\lambda}, \mathrm{P}_{\lambda}\right]$ and eighteen subintervals of $\left[-\mathrm{P}_{\lambda}, \mathrm{P}_{\lambda}\right]$ are either mapped to $\left[0, \mathrm{P}_{\lambda}\right]$ or $\left[-\mathrm{P}_{\lambda}, 0\right]$.

If En denotes the union of all open subintervals escaping under the $\mathrm{n}^{\text {th }}$ iteration of $\mathrm{f}$, we see that $\mathrm{E}_{1}=(-\mathrm{s},-\mathrm{r}) \cup(\mathrm{r}, \mathrm{s})$ and

$\mathrm{E}_{2}=\left(\mathrm{s}_{1}, \mathrm{r}_{1}\right) \cup\left(\mathrm{r}_{2}, \mathrm{~s}_{2}\right) \cup\left(\mathrm{s}_{3}, \mathrm{r}_{3}\right) \cup\left(\mathrm{r}_{1}{ }^{\prime}, \mathrm{s}_{1}{ }^{\prime}\right) \cup\left(\mathrm{s}_{2}{ }^{\prime}, \mathrm{r}_{2}{ }^{\prime}\right) \cup\left(\mathrm{r}_{3}{ }^{\prime}, \mathrm{s}_{3}{ }^{\prime}\right)$, where $\mathrm{r}_{1}, \mathrm{r}_{2}, \mathrm{r}_{3}$ are the three roots of $x^{3}+\lambda x+r=0$,

$r_{1}{ }^{\prime}, r_{2}{ }^{\prime}, r_{3}{ }^{\prime}$ are the three roots of $x^{3}+\lambda x-r=0$,

$\mathrm{s}_{1}, \mathrm{~s}_{2}, \mathrm{~s}_{3}$ are the three roots of $\mathrm{x}^{3}+\lambda \mathrm{x}+\mathrm{s}=0$ and

$\mathrm{s}_{1}{ }^{\prime}, \mathrm{s}_{2}{ }^{\prime}, \mathrm{s}_{3}{ }^{\prime}$ are the three roots of $\mathrm{x}^{3}+\lambda \mathrm{x}-\mathrm{s}=0$.

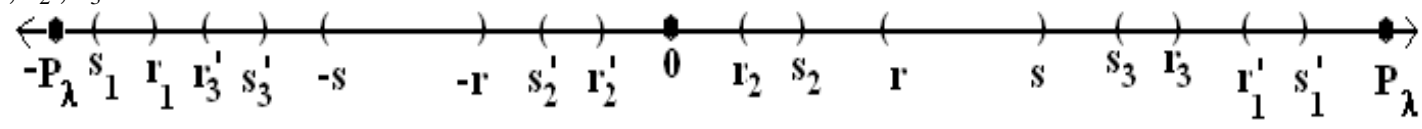

In general, En $=$ the union of $2\left(3^{\mathrm{n}-1}\right)$ escaping open intervals.

Then $\mathrm{E}=\bigcup_{n=1}^{\infty} \mathrm{En}$ is the set of all points which escape from $\left[-\mathrm{P}_{\lambda}, \mathrm{P}_{\lambda}\right]$ under all the iterations of $\mathrm{f}$. And let Fn be the union of all subintervals of $\left[-\mathrm{P}_{\lambda}, \mathrm{P}_{\lambda}\right]$ which are closed and remain in $\left[-\mathrm{P}_{\lambda}, \mathrm{P}_{\lambda}\right]$ after the ${ }^{\text {th }}$ iteration of f. Then,

$\mathrm{F}_{1}=\left[-\mathrm{P}_{\lambda},-\sqrt{-\lambda}\right] \cup[-\sqrt{-\lambda},-\mathrm{s}] \cup[-\mathrm{r}, 0] \cup[0, \mathrm{r}] \cup[\mathrm{s}, \sqrt{-\lambda}] \cup\left[\sqrt{-\lambda}, \mathrm{P}_{\lambda}\right]$

$\mathrm{F}_{2}=$ the union of 18 closed subintervals -9 pairs of adjacent intervals, each pair having one ending point in common.

In general, Fn $=$ the union of $6\left(3^{\mathrm{n}-1}\right)$ closed subintervals $-3^{\mathrm{n}}$ pairs of adjacent intervals, each pair having one ending point in common.

Let $\mathrm{F}=\bigcap_{n=1}^{\infty} \mathrm{Fn}$. Then $\mathrm{F}$ is the set of points in $\mathrm{R}$ which always remain inside $\left[-\mathrm{P}_{\lambda}, \mathrm{P}_{\lambda}\right]$ under the iterations of $\mathrm{f}$.

It is interesting to note that this set $\mathrm{F}$ possesses some nice topological and fractal-like properties, which is not our area of focus now. Since dynamics of $\mathrm{f}$ on $\mathrm{E}$ as well as on $\mathrm{R}-\left[-\mathrm{P}_{\lambda}, \mathrm{P}_{\lambda}\right]$ is simple enough, it remains to understand the dynamics of $\mathrm{f}$ on $\mathrm{F}$ to complete the discussions of the dynamics of $\mathrm{f}(\mathrm{x})=\mathrm{x}^{3}+\lambda \mathrm{x}$ for $\lambda<-3$.

First we explore some details about F : We can see that Fn is a union of closed intervals Fn,k such that Fn,k and $\mathrm{Fn}, \mathrm{k}+1, \quad \mathrm{k}=1,3 \ldots \ldots, 6\left(3^{\mathrm{n}-1}\right)-1$, of these subintervals are always adjacent in the sense that they have one end point in common. Except these couples, the other intervals are mutually disjoint. For example, let us look at $\mathrm{F}_{1}$. 


\section{$\underline{\text { Subintervals of }\left[-\mathrm{P}_{\lambda} \mathrm{P}_{\lambda}\right] \text { at the end of first iteration }}$}

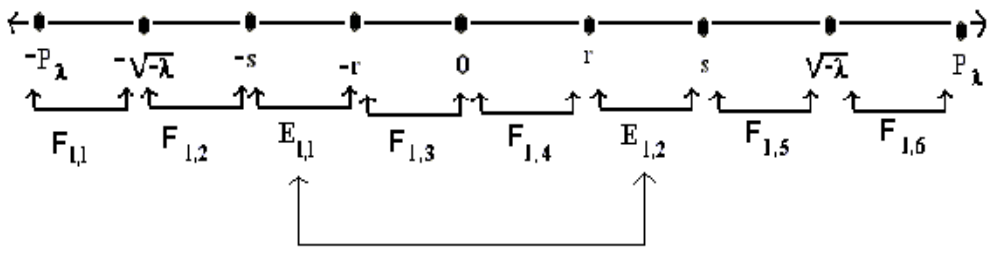

Escaping subintervals

We can see that $F_{1}=F_{1,1} \cup F_{1,2} \cup F_{1,3} \cup F_{1,4} \cup F_{1,5} \cup F_{1,6}$. We can see that two subintervals Fn,k and Fn,k+1, $\mathrm{k}=1,3 \ldots \ldots, 6\left(3^{\mathrm{n}-1}\right)-1$ in $\mathrm{F}_{1}$ are adjacent, but no other subintervals in $\mathrm{F}_{1}$ are adjacent. In the above figure, $\mathrm{F}_{1,1}$, $F_{1,2} F_{1,3}, F_{1,4}, F_{1,5}$ and $F_{1,6}$ are remaining intervals (which are mapped either to $\left[0, P_{\lambda}\right]$ or $\left[-P_{\lambda}, 0\right]$ at the first iteration) and $\mathrm{E}_{1,1}, \mathrm{E}_{1,2}$ are the intervals escaping out of $\left[-\mathrm{P}_{\lambda}, \mathrm{P}_{\lambda}\right]$ at the first iteration.

\section{$\underline{\text { Subintervals of }\left[-\mathrm{P}_{\lambda}, \mathrm{P}_{\lambda}\right] \text { at the end of second iteration }}$}

The following two figures show that similar thing happens in $\mathrm{F}_{2}$.

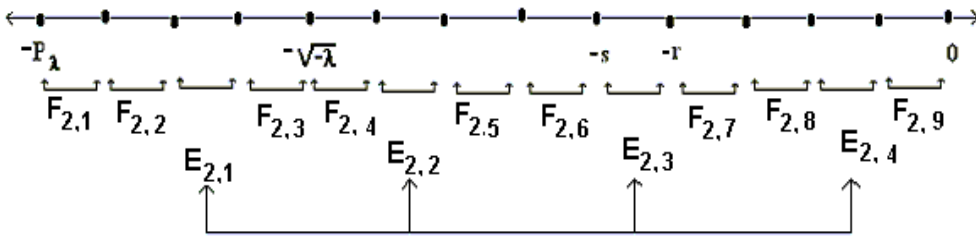

Escaping intervals

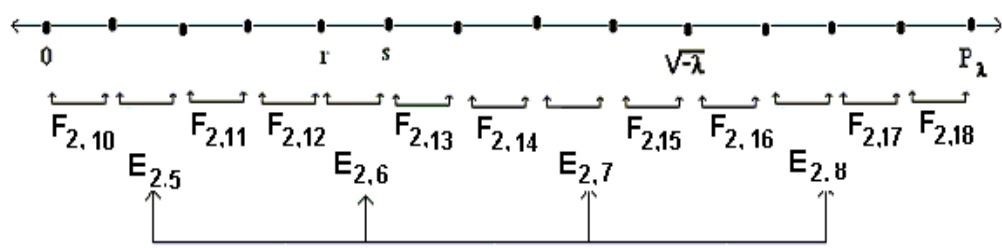

Escaping intervals

Here $F_{2}=F_{2,1} \cup F_{2,2} \cup F_{2,3} \cup F_{2,4} \cup F_{2,5} \cup F_{2,6} \cup F_{2,7} \cup F_{2,8} \cup F_{2,9} \cup F_{2,10} \cup F_{2,11} \cup F_{2,12} \cup F_{2,13} \cup F_{2,14} \cup F_{2,15}$ $\cup \mathrm{F}_{2,16} \cup \mathrm{F}_{2,17} \cup \mathrm{F}_{2,18}$.

Escaping intervals are : $E_{2,1}, E_{2,2}, E_{2,3}, E_{2,4}, E_{2,5}, E_{2,6}, E_{2,7}, E_{2,8}$.

Thus after every two remaining intervals, an escaping interval appears.

We summarize this discussion in the following statement :

Theorem $2.1:$ For the function $\mathrm{f}:\left[-\mathrm{P}_{\lambda}, \mathrm{P}_{\lambda}\right] \rightarrow \mathrm{R}, \mathrm{f}(\mathrm{x})=\mathrm{x}^{3}+\lambda \mathrm{x}, \lambda<-3$, there exists the domain of chaos $\mathrm{F}=\bigcap_{n=1}^{\infty} \mathrm{Fn} \quad$ such that $\mathrm{f}: \mathrm{F} \rightarrow \mathrm{F}$ is onto ; and further there exists escaping open set $\mathrm{E}=\bigcup_{n=1}^{\infty} \mathrm{En}$ in $\left[-\mathrm{P}_{\lambda}, \mathrm{P}_{\lambda}\right]$ such that $\mathrm{F}$ is the boundary of $\mathrm{E}$.

- $\quad$ Now we prove the following useful result :

Theorem 2.2: $\quad$ For $\lambda<-3$, length of the subintervals in Fn tends to 0 as $n \rightarrow \infty$.

Proof : $\quad$ We recall the following observations from the construction of $F$ :

If $\mathrm{F}_{0}=\left[-\mathrm{P}_{\lambda}, \mathrm{P}_{\lambda}\right]$, then $\mathrm{F}_{1}$ is the union of the six closed intervals which map inside $\left[-\mathrm{P}_{\lambda}, \mathrm{P}_{\lambda}\right]$ under the first iteration of $f$.

So, $\mathrm{F}_{1}=\left[-\mathrm{P}_{\lambda},-\sqrt{-\lambda}\right] \cup[-\sqrt{-\lambda},-\mathrm{s}] \cup[-\mathrm{r}, 0] \cup[0, \mathrm{r}] \cup[\mathrm{s}, \sqrt{-\lambda}] \cup\left[\sqrt{-\lambda}, \mathrm{P}_{\lambda}\right]$.

Now each subinterval of $F_{1}$ is divided into four more subintervals such that one of these subinterval is mapped outside $\left[-\mathrm{P}_{\lambda}, \mathrm{P}_{\lambda}\right]$ and each of the other three subintervals is mapped to one of the subintervals of $\mathrm{F}_{1}$.

Thus we can see that every subinterval Fn, $i$ of $F n$ is contained in some subinterval of $F_{n-1}$, say $F_{n-1, j}$. So if $F n, i=\left[a_{n}, b_{n}\right]$ and $F_{n-1, j}=\left[a_{n-1}, b_{n-1}\right]$, then $\left|a_{n}-b_{n}\right|<\left|a_{n-1}-b_{n-1}\right|$. 
So, $\mathrm{k}_{1}\left|\mathrm{a}_{\mathrm{n}}-\mathrm{b}_{\mathrm{n}}\right|=\left|\mathrm{a}_{\mathrm{n}-1}-\mathrm{b}_{\mathrm{n}-1}\right|$ for some $\mathrm{k}_{1}>1$.

By the same argument, $\mathrm{k}_{2}\left|\mathrm{a}_{\mathrm{n}-1}-\mathrm{b}_{\mathrm{n}-1}\right|=\left|\mathrm{a}_{\mathrm{n}-2}-\mathrm{b}_{\mathrm{n}-2}\right|$ for some $\mathrm{k}_{2}>1$.

So, $k_{1} k_{2}\left|a_{n}-b_{n}\right|=\left|a_{n-2}-b_{n-2}\right|$ for $k_{1}, k_{2}>1$.

Continuing this way, $\mathrm{k}_{1} \mathrm{k}_{2} \mathrm{k}_{3} \ldots \ldots \mathrm{k}_{\mathrm{n}}\left|\mathrm{a}_{\mathrm{n}}-\mathrm{b}_{\mathrm{n}}\right|=\left|\mathrm{a}_{0}-\mathrm{b}_{0}\right|$ for $\mathrm{k}_{1}, \mathrm{k}_{2} \ldots \mathrm{k}_{\mathrm{n}}>1$. But $\left[\mathrm{a}_{0}, \mathrm{~b}_{0}\right]$ is either $\left[0, \mathrm{P}_{\lambda}\right]$ or $\left[-\mathrm{P}_{\lambda}, 0\right]$, and so, $\left|\mathrm{a}_{0}-\mathrm{b}_{0}\right|=\mathrm{P}_{\lambda}$.

So, $\mathrm{k}_{1} \mathrm{k}_{2} \mathrm{k}_{3} \ldots . . \mathrm{k}_{\mathrm{n}}\left|\mathrm{a}_{\mathrm{n}}-\mathrm{b}_{\mathrm{n}}\right|=\mathrm{P}_{\lambda}$.

If $\mathrm{k}=\operatorname{mini}\left\{\mathrm{k}_{\mathrm{i}} / \mathrm{i}=1,2,3 \ldots \ldots, \mathrm{n}\right\}$, then $\mathrm{k}>1$ and $\mathrm{k}_{1} \mathrm{k}_{2} \mathrm{k}_{3} \ldots . . \mathrm{k}_{\mathrm{n}}>\mathrm{k}^{\mathrm{n}}$.

So, $\mathrm{k}^{\mathrm{n}}\left|\mathrm{a}_{\mathrm{n}}-\mathrm{b}_{\mathrm{n}}\right|<\mathrm{k}_{1} \mathrm{k}_{2} \mathrm{k}_{3} \ldots . . \mathrm{k}_{\mathrm{n}}\left|\mathrm{a}_{\mathrm{n}}-\mathrm{b}_{\mathrm{n}}\right|=\mathrm{P}_{\lambda}$.

Hence, $\left|a_{n}-b_{n}\right|<P_{\lambda} / k^{n}$ for $k>1$.

Thus length of a subinterval in Fn tends to zero as $\mathrm{n} \rightarrow \infty$.

- We can work out an another proof which works for most of the values of $\lambda$ in the left of -3 . The result is as follows :

- Theorem 2.3: $\quad$ For $\lambda<-1-\frac{3}{\sqrt{2}}(\approx-3.121320344)$., length of the subintervals in Fn tends to 0 as $\mathrm{n} \rightarrow \infty$.

Proof: Let $\lambda<-1-\frac{3}{\sqrt{2}}(\approx-3.121320344)$. Then we claim that $\left|f^{\prime}(x)\right|>1$ for all $x \in F_{1}=\left[-P_{\lambda}, P_{\lambda}\right]-$ $\{(\mathrm{r}, \mathrm{s}) \cup(-\mathrm{s},-\mathrm{r})\}$

Since $\mathrm{f}(\mathrm{x})=\mathrm{x}^{3}+\lambda \mathrm{x} \Rightarrow \mathrm{f}^{\prime \prime}(\mathrm{x})=6 \mathrm{x}>0$ for $\mathrm{x}>0$. So, $\mathrm{f}^{\prime}$ is an increasing function for $\lambda>0$.

Next, $\lambda=-1-\frac{3}{\sqrt{2}} \Rightarrow \mathrm{f}^{\prime}(\mathrm{r})=3 \mathrm{r}^{2}+\lambda=3 \mathrm{r}^{2}+\left(-1-\frac{3}{\sqrt{2}}\right)=-1$.

Now $r=\frac{\sqrt{1-\lambda}}{2}-\frac{\sqrt{-3-\lambda}}{2} \Rightarrow \frac{d r}{d \lambda}=\frac{1}{4}\left[\frac{1}{\sqrt{-3-\lambda}}-\frac{1}{\sqrt{1-\lambda}}\right]>0$ for $\lambda<-3$.

So, $r$ decreases as $\lambda$ decreases. Hence, $f^{\prime}(r)=3 r^{2}+\lambda$ decreases as $\lambda$ decreases. So by (2), $\quad \lambda<-1-\frac{3}{\sqrt{2}}$ $\Rightarrow \mathrm{f}^{\prime}(\mathrm{r})<-1$; and by $(1), \mathrm{f}^{\prime}(\mathrm{x})<-1$ for all $\mathrm{x} \in[0, \mathrm{r}]$, i.e., $\left|\mathrm{f}^{\prime}(\mathrm{x})\right|>1$ for all $\mathrm{x} \in[0, \mathrm{r}]$.

Similarly, $\quad \lambda=\frac{-1-3 \sqrt{3}}{2}(\approx-3.098076211)$

$\Rightarrow \mathrm{f}^{\prime}(\mathrm{s})=3 \mathrm{~s}^{2}+\lambda=3 \mathrm{~s}^{2}+\left(\frac{-1-3 \sqrt{3}}{2}\right)=1$

Now $s=\frac{\sqrt{1-\lambda}}{2}+\frac{\sqrt{-3-\lambda}}{2} \Rightarrow \frac{d s}{d \lambda}=\frac{1}{4}\left[\frac{-1}{\sqrt{-3-\lambda}}-\frac{1}{\sqrt{1-\lambda}}\right]<0$ for $\lambda<-3$.

So, $\mathrm{s}$ increases as $\lambda$ decreases. Hence, $\mathrm{f}^{\prime}(\mathrm{s})=3 \mathrm{~s}^{2}+\lambda$ increases as $\lambda$ decreases. So by (3), $\lambda<\frac{-1-3 \sqrt{3}}{2}$ $\Rightarrow \mathrm{f}^{\prime}(\mathrm{s})>1$; and by $(1), \mathrm{f}^{\prime}(\mathrm{x})>1$ for all $\mathrm{x} \in\left[\mathrm{s}, \mathrm{P}_{\lambda}\right]$, i.e. $\left|\mathrm{f}^{\prime}(\mathrm{x})\right|>1$ for all $\mathrm{x} \in\left[\mathrm{s}, \mathrm{P}_{\lambda}\right]$.

Since $-1-\frac{3}{\sqrt{2}}<\frac{-1-3 \sqrt{3}}{2}$ for $\lambda<-3$, from (A) and (B),

$\lambda<-1-\frac{3}{\sqrt{2}} \Rightarrow\left|\mathrm{f}^{\prime}(\mathrm{x})\right|>1$ for all $\mathrm{x} \in\left[0, \mathrm{P}_{\lambda}\right]-(\mathrm{r}, \mathrm{s})$.

Since $\mathrm{f}^{\prime}(-\mathrm{x})=3(-\mathrm{x})^{2}+\lambda=3(\mathrm{x})^{2}+\lambda=\mathrm{f}^{\prime}(\mathrm{x})$, we have,

$\lambda<-1-\frac{3}{\sqrt{2}} \Rightarrow\left|\mathrm{f}^{\prime}(\mathrm{x})\right|>1$ for all $\mathrm{x} \in\left[-\mathrm{P}_{\lambda}, 0\right]-(-\mathrm{s},-\mathrm{r})$.

Hence, $\left|\mathrm{f}^{\prime}(\mathrm{x})\right|>1$ for all $\mathrm{x} \in \mathrm{F}_{1}=\left[-\mathrm{P}_{\lambda}, \mathrm{P}_{\lambda}\right]-\{(\mathrm{r}, \mathrm{s}) \cup(-\mathrm{s},-\mathrm{r})\}$.

So, there exists some $\mathrm{k}>1$ such that $\left|\mathrm{f}^{\prime}(\mathrm{x})\right|>\mathrm{k}>1$ for all $\mathrm{x} \in \mathrm{F}_{1}=\left[-\mathrm{P}_{\lambda}, \mathrm{P}_{\lambda}\right]-\{(\mathrm{r}, \mathrm{s}) \cup(-\mathrm{s},-\mathrm{r})\}$. 
We now show that length of the subintervals in Fn tends to 0 as $n \rightarrow \infty$.

Let $[\mathrm{a}, \mathrm{b}]$ be any subinterval in Fn. Then $\mathrm{f}^{\mathrm{n}}$ maps $[\mathrm{a}, \mathrm{b}]$ with the endpoints of $[\mathrm{a}, \mathrm{b}]$ mapped to 0 and $\mathrm{P}_{\lambda}$ $\left(\right.$ or $\left.-\mathrm{P}_{\lambda}\right)$.

Hence, $\left|\mathrm{f}^{\mathrm{n}}(\mathrm{b})-\mathrm{f}^{\mathrm{n}}(\mathrm{a})\right|=\mathrm{P}_{\lambda}$.

Now $f$ being continuous on $[a, b]$, by the mean value theorem,

$\begin{aligned}|\mathrm{f}(\mathrm{b})-\mathrm{f}(\mathrm{a})| & =|\mathrm{b}-\mathrm{a}|\left|\mathrm{f}^{\prime}(\mathrm{c})\right| \text {, for some } \mathrm{c} \in(\mathrm{a}, \mathrm{b}) . \\ & >\mathrm{k}|\mathrm{b}-\mathrm{a}|(\text { by (4) })\end{aligned}$

Again, $\mathrm{f}^{2}$ being continuous on $[\mathrm{a}, \mathrm{b}]$, by the mean value theorem for $\mathrm{f}^{2}$ on $[\mathrm{a}, \mathrm{b}]$,

$$
\begin{aligned}
& \left|f^{2}(b)-f^{2}(a)\right|=|f(b)-f(a)|\left|\left(f^{2}\right)^{\prime}(d)\right| \text {, for some } d \in(a, b) \text {. } \\
& >\quad \mathrm{k}|\mathrm{b}-\mathrm{a}| \cdot \mathrm{k} \quad \text { (by (5) and the fact that } \\
& =\quad \mathrm{k}^{2} \cdot|\mathrm{b}-\mathrm{a}| \\
& \left.\left|\left(f^{2}\right)^{\prime}(\mathrm{d})\right|=\left|\mathrm{f}^{\prime}(\mathrm{d})\right|\left|\mathrm{f}^{\prime}(\mathrm{f}(\mathrm{d}))\right|>1 . \mathrm{k}\right)
\end{aligned}
$$

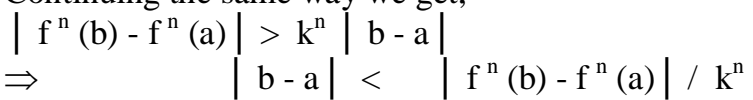

$$
\begin{aligned}
& \text { But } \quad\left|f^{n}(b)-f^{n}(a)\right|=P_{\lambda} \text {. } \\
& \Rightarrow \quad \mid \begin{array}{l}
\mathrm{b}-\mathrm{a} \mid \\
\text { So, as } \mathrm{n} \rightarrow \infty,|\mathrm{b}-\mathrm{a}| \rightarrow 0 \text {. }
\end{array}
\end{aligned}
$$

Finally, we move to the main result of the paper, showing that $f(x)=x^{3}+\lambda x, \lambda<-3$, is chaotic on $F$. By the popular definition of a chaotic function by R. L. Devaney [2], a real function $f$ on set $F$ is chaotic if

a) f has sensitive dependence on initial conditions on $F$.

b) $\quad f$ is topologically transitive on $F$ and

c) periodic points of $f$ are dense in $F$.

However the following result by Banks, Brooks, Cairns, David and Stacey [4] suggests that under some circumstances, if $\mathrm{f}$ satisfies the conditions of topological transitivity and density of periodic points, then $\mathrm{f}$ is chaotic, i.e. the sensitivity dependence follows automatically if the other two conditions are satisfied. The result is as follows :

Theorem 2.4 : Let $\mathrm{D}$ be an infinite subset of real numbers and $\mathrm{f}: \mathrm{D} \rightarrow \mathrm{D}$ is continuous. If $\mathrm{f}$ is topologically transitive on $\mathrm{D}$ and the periodic points of $\mathrm{f}$ are dense in $\mathrm{D}$, then $\mathrm{f}$ is chaotic on $\mathrm{D}$.

Since our set $F$ is an infinite subset of real numbers and $f: F \rightarrow F$ is continuous, we only need to show the conditions of topological transitivity and density of periodic points, to show that $\mathrm{f}(\mathrm{x})=\mathrm{x}^{3}+\lambda \mathrm{x}, \quad \lambda<-3$, is chaotic on F. However, after completing the proof, we shall also work out that $f(x)=x^{3}+\lambda x, \lambda<-3$, has a sensitivity dependence on initial conditions on F. During the proof, we shall also use the following simple result about the fixed points :

Proposition 2.1: Let I be a closed interval and $f: I \rightarrow R$ be a continuous function. If $I \subset f(I)$, then $f$ has a fixed point in $\mathrm{I}$.

And now the main result follows :

Theorem 2.5: $\mathrm{f}(\mathrm{x})=\mathrm{x}^{3}+\lambda \mathrm{x}, \lambda<-3$, is chaotic on $\mathrm{F}$.

Proof : To show that $\mathrm{f}(\mathrm{x})=\mathrm{x}^{3}+\lambda \mathrm{x}, \lambda<-3$, is chaotic on $\mathrm{F}$, we shall show :

a) $\quad f$ is topologically transitive on $F$ and

b) periodic points of $f$ are dense in $F$.

a) To show that the function $\mathrm{f}: \mathrm{F} \rightarrow \mathrm{F}, \mathrm{F} \subset \mathrm{R}$ is topologically transitive, we should show that for any pair of nonempty open sets $U, V$ in $F$, there exist some $k>0$ such that $f^{k}(U) \cap V \neq \phi$.

Let $U$ and $V$ be any two nonempty open sets in $F$. Then there exist nonempty open sets $U^{\prime}$ and $V^{\prime}$ in $\left[-P_{\lambda}, P_{\lambda}\right]$ such that $U=U^{\prime} \cap F$ and $V=V^{\prime} \cap F$. Since $U=U^{\prime} \cap F \neq \phi$, $U^{\prime}$ contains at least one point of $F$, say $x$. $\mathrm{U}^{\prime}$ being open in $\left[-\mathrm{P}_{\lambda}, \mathrm{P}_{\lambda}\right]$, there exists some $\varepsilon>0$ such that $(\mathrm{x}-\varepsilon, \mathrm{x}+\varepsilon)$ is contained in $\mathrm{U}^{\prime}$.

As $x \in F, \quad x \in \bigcap_{n=1}^{\infty} F n$, and hence, $x \in F$ Fn for each $n$. Since the length of each of the subintervals in Fn tends to 0 as $\mathrm{n} \rightarrow \infty$, there exists some $\mathrm{m}$ such that the length of the subintervals in Fm is less than $\varepsilon$. Let $\mathrm{Fm}, \mathrm{k}$ be a subinterval of Fm which contains $\mathrm{x}$. Then Fm, $\mathrm{k}$ is a subset of $\mathrm{U}^{\prime}$ and contains $\mathrm{x}$. Hence, Fm, $\mathrm{k} \cap \mathrm{F}$ is a subset of $\mathrm{U}$, containing $\mathrm{x}$.

Now $\mathrm{f}^{\mathrm{m}}$ maps $\mathrm{Fm}, \mathrm{k}$ onto $\left[0, \mathrm{P}_{\lambda}\right]$ or $\left[-\mathrm{P}_{\lambda}, 0\right]$. Suppose $\mathrm{f}^{\mathrm{m}}$ maps $\mathrm{Fm}, \mathrm{k}$ onto $\left[0, \mathrm{P}_{\lambda}\right]$. ( similar proof holds if $\mathrm{f}^{\mathrm{m}}$ maps Fm,k onto [ $\left.-\mathrm{P}_{\lambda}, 0\right]$.)

If $\mathrm{V} \cap\left[0, \mathrm{P}_{\lambda}\right] \neq \phi$, then there exists some $\mathrm{y}$ in $\mathrm{V} \cap\left[0, \mathrm{P}_{\lambda}\right]$. Since $\mathrm{y} \in \mathrm{V}, \mathrm{y} \in \mathrm{F}$. Now since $\mathrm{y} \in$ $\left[0, P_{\lambda}\right]$, there exists some $x$ in $F m, k$ such that $f^{m}(x)=y$. And since $y \in F, f^{n}(y) \in F$ for all $n$. Therefore $\mathrm{f}^{\mathrm{n}}(\mathrm{x}) \in \mathrm{F}$ for all $\mathrm{n}$. Thus, $\mathrm{x}$ must be in $\mathrm{F}$ because $\mathrm{y} \in \mathrm{F}$. So, $\mathrm{x} \in \mathrm{U}$ and $\mathrm{f}^{\mathrm{m}}(\mathrm{x})=\mathrm{y} \in \mathrm{V}$.

Hence, $f^{\mathrm{m}}(\mathrm{U}) \cap \mathrm{V} \neq \phi$. 
If $\mathrm{V} \cap\left[0, \mathrm{P}_{\lambda}\right]=\phi$, then we must have $\mathrm{V} \cap\left[-\mathrm{P}_{\lambda}, 0\right] \neq \phi$. (otherwise $\mathrm{V}=\phi$.) Since $\mathrm{f}^{\mathrm{m}}$ maps $\mathrm{Fm}, \mathrm{k}$ onto $\left[0, \mathrm{P}_{\lambda}\right]$ and $0<\mathrm{r}<\mathrm{P}_{\lambda}$, a subinterval of Fm,k is mapped to $[0, \mathrm{r}]$. But $\quad[0, \mathrm{r}]$ is mapped to $\left[-\mathrm{P}_{\lambda}, 0\right]$ by the next iteration of $\mathrm{f}$.

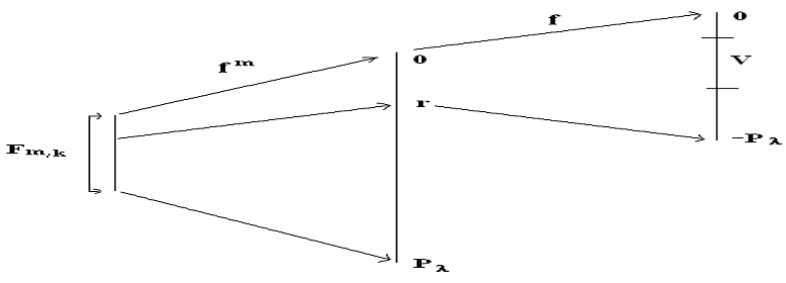

Hence, a subinterval of Fm,k is mapped to $\left[-\mathrm{P}_{\lambda}, 0\right]$ under $\mathrm{f}^{\mathrm{m}+1}$. Therefore, $\mathrm{f}^{\mathrm{m}+1}(\mathrm{Fm}, \mathrm{k}) \cap \mathrm{V} \neq \phi$, and hence, $\mathrm{f}^{\mathrm{m}+1}(\mathrm{U}) \cap \mathrm{V} \neq \phi$.

b) To prove that periodic points of $\mathrm{f}$ are dense in $\mathrm{F}$, we need to show that any open set in $\mathrm{F}$ contains a periodic point of $\mathrm{f}$.

Let $U$ be an open set in F. As seen in part (a) above, there exists a subinterval Fm,k such that $\mathrm{Fm}, \mathrm{k} \cap \mathrm{F} \subset \mathrm{U}$. As seen during the construction of set $\mathrm{F}, \mathrm{Fm}, \mathrm{k}$ is divided into four subintervals of itself such that one of the subintervals, say $\mathrm{I}_{1}$, is mapped onto $\quad\left[0, \mathrm{P}_{\lambda}\right]$ under $\mathrm{f}^{\mathrm{m}+1}$ and one other subinterval, say $\mathrm{I}_{2}$, is mapped onto $\left[-\mathrm{P}_{\lambda}, 0\right]$ under $\mathrm{f}^{\mathrm{m}+1}$. Thus, $\mathrm{f}^{\mathrm{m}+1}\left(\mathrm{I}_{1}\right)=\left[0, \mathrm{P}_{\lambda}\right]$ and $\mathrm{f}^{\mathrm{m}+1}\left(\mathrm{I}_{2}\right)=\left[-\mathrm{P}_{\lambda}, 0\right]$.

Now, Fm,k is a subinterval in Fm, and hence either Fm, $\mathrm{k} \subset\left[0, \mathrm{P}_{\lambda}\right]$ or $\mathrm{Fm}, \mathrm{k} \subset\left[-\mathrm{P}_{\lambda}, 0\right]$.

If Fm,k $\subset\left[0, \mathrm{P}_{\lambda}\right]$, then $\mathrm{I}_{1} \subset \mathrm{Fm}, \mathrm{k} \subset\left[0, \mathrm{P}_{\lambda}\right]=\mathrm{f}^{\mathrm{m}+1}\left(\mathrm{I}_{1}\right)$. So, $\mathrm{f}^{\mathrm{m}+1}$ has a fixed point, say p, in $\mathrm{I}_{1} \subset \mathrm{Fm}, \mathrm{k}$. Thus, $\mathrm{p} \in \mathrm{Fm}, \mathrm{k} \cap \mathrm{F} \subset \mathrm{U}$.

If Fm,k $\subset\left[-P_{\lambda}, 0\right]$, then $I_{2} \subset F m, k \subset\left[-P_{\lambda}, 0\right]=f^{m+1}\left(I_{2}\right)$. So, $f^{m+1}$ has a fixed point, say q, in $I_{2} \subset$ Fm,k. Thus, $\mathrm{q} \in \mathrm{Fm}, \mathrm{k} \cap \mathrm{F} \subset \mathrm{U}$.

Hence, an arbitrary open set $\mathrm{U}$ in $\mathrm{F}$ contains a periodic point of $\mathrm{f}$. This completes the proof.

Note : Though we don't need the condition of sensitivity dependence on initial conditions to show that $\mathrm{f}(\mathrm{x})=\mathrm{x}^{3}+\lambda \mathrm{x}, \lambda<-3$, is chaotic on $\mathrm{F}$, we can work out the proof to show that $\mathrm{f}(\mathrm{x})=\mathrm{x}^{3}+\lambda \mathrm{x}, \lambda<-3$, has sensitivity dependence on initial conditions on $\mathrm{F}$. The proof is as follows :

By the definition of the sensitivity dependence on initial conditions for a function $\mathrm{f}$, we need to show that there exists some $\delta>0$ such that for any $\mathrm{x} \in \mathrm{F}$ and any neighbourhood $\mathrm{N}$ of $\mathrm{x}$, there exists some $\mathrm{y} \in \mathrm{F}$ and $\mathrm{n} \geq 0$ such that $\left|\mathrm{f}^{\mathrm{n}}(\mathrm{x})-\mathrm{f}^{\mathrm{n}}(\mathrm{y})\right|>\delta$.

Let $\delta=\mathrm{P}_{\lambda} / 2$. Let $\mathrm{x} \in \mathrm{F}$ and $\mathrm{N}^{\prime}=(\mathrm{x}-\varepsilon, \mathrm{x}+\varepsilon)$ be a neighbourhood of $\mathrm{x}$ in $\mathrm{R}$. Then $\mathrm{N}=\mathrm{N}^{\prime} \cap \mathrm{F}$ is a neighbourhood of $x$ in $F$.

Since $\mathrm{x} \in \mathrm{F}, \mathrm{x} \in \bigcap_{n=1}^{\infty} \mathrm{Fn}$, and hence, $\mathrm{x} \in \mathrm{Fn}$ for each $\mathrm{n}$. As length of each of the subintervals in Fn tends to 0 as $\mathrm{n} \rightarrow \infty$, there exists some $\mathrm{m}$ such that the length of the subintervals in Fm is less than $\varepsilon$. Let Fm,k be a subinterval of Fm which contains $\mathrm{x}$. Then Fm,k is a subset of $\mathrm{N}^{\prime}$ and contains $\mathrm{x}$. Hence, Fm,k $\cap \mathrm{F}$ is a subset of $\mathrm{N}$, containing $\mathrm{x}$.

Now $\mathrm{f}^{\mathrm{m}}$ maps $\mathrm{Fm}, \mathrm{k}$ onto $\left[0, \mathrm{P}_{\lambda}\right]$ or $\left[-\mathrm{P}_{\lambda}, 0\right]$.

Suppose $\mathrm{f}^{\mathrm{m}}$ maps $\mathrm{Fm}, \mathrm{k}$ onto $\left[0, \mathrm{P}_{\lambda}\right]$.

Then some point of Fm,k, say p, is mapped to 0 and some other point of Fm,k, say q, is mapped to $P_{\lambda}$ under $\mathrm{f}^{\mathrm{m}}$. Since, $\mathrm{p}, \mathrm{q}$ remain in $\left[-\mathrm{P}_{\lambda}, \mathrm{P}_{\lambda}\right]$ under the iterations of $\mathrm{f}, \mathrm{p}, \mathrm{q} \in \mathrm{F}$. Thus, $\mathrm{p}, \mathrm{q} \in \mathrm{Fm}, \mathrm{k} \cap \mathrm{F}$, i.e., $\mathrm{p}, \mathrm{q} \in \mathrm{N}$. Since $\mathrm{x} \in \mathrm{Fm}, \mathrm{k}, \mathrm{f}^{\mathrm{m}}(\mathrm{x}) \in\left[0, \mathrm{P}_{\lambda}\right]$, but $\mathrm{f}^{\mathrm{m}}(\mathrm{x}) \neq \mathrm{P}_{\lambda} / 2$ because $\mathrm{P}_{\lambda} / 2$ lies in the interval $(\mathrm{r}, \mathrm{s})$ and points of $(\mathrm{r}, \mathrm{s})$ cannot be in $\mathrm{F}$ as they escape from $\left[-\mathrm{P}_{\lambda}, \mathrm{P}_{\lambda}\right]$ under $\mathrm{f}$.

So, $\mathrm{f}^{\mathrm{m}}(\mathrm{x}) \in\left[0, \mathrm{P}_{\lambda} / 2\right)$ or $\mathrm{f}^{\mathrm{m}}(\mathrm{x}) \in\left(\mathrm{P}_{\lambda} / 2, \mathrm{P}_{\lambda}\right]$.

If $\mathrm{f}^{\mathrm{m}}(\mathrm{x}) \in\left[0, \mathrm{P}_{\lambda} / 2\right)$, then take $\mathrm{q}=\mathrm{y}$. So, $\mathrm{y} \in \mathrm{N}$ with $\mathrm{f}(\mathrm{y})=\mathrm{P}_{\lambda}$. Hence, $\left|\mathrm{f}^{\mathrm{m}}(\mathrm{x})-\mathrm{f}^{\mathrm{m}}(\mathrm{y})\right|>\delta\left(=\mathrm{P}_{\lambda} / 2\right)$.

Similarly, If $\mathrm{f}^{\mathrm{m}}(\mathrm{x}) \in\left(\mathrm{P}_{\lambda} / 2, \mathrm{P}_{\lambda}\right]$ then take $\mathrm{p}=\mathrm{y}$. So, $\mathrm{y} \in \mathrm{N}$ with $\mathrm{f}(\mathrm{y})=0$.

Hence, $\quad\left|\mathrm{f}^{\mathrm{m}}(\mathrm{x})-\mathrm{f}^{\mathrm{m}}(\mathrm{y})\right|>\delta\left(=\mathrm{P}_{\lambda} / 2\right)$.

Similar proof holds if $\mathrm{f}^{\mathrm{m}}$ maps $\mathrm{Fm}, \mathrm{k}$ onto $\left[-\mathrm{P}_{\lambda}, 0\right]$.

Thus, the dynamics of $\mathrm{f}(\mathrm{x})=\mathrm{x}^{3}+\lambda \mathrm{x}$ is very interesting for $\lambda<-3$ and it suggests that the dynamics of simple polynomial functions can be very complex.

\section{Conclusion}

A real quadratic family [2] $\mathrm{f}(\mathrm{x})=\mathrm{kx}(1-\mathrm{x}), \mathrm{k} \in \mathrm{R}$ has indicated that even the simplest looking functions may have the complex dynamics. This paper has aimed to study the behavior of cubic family functions and its chaotic behavior. The dynamics of cubic family is more complex and this family of functions do exhibit the topological transitivity, sensitivity dependence on initial conditions and the density of periodic points, which 
Fractal Trap For The Chaotic Behavior Of Real Cubic Polynomials

are the essential ingredients of the chaotic behavior. This realization about cubic family has opened up a direction towards the generalization of the chaotic behavior of the real polynomials.

\section{References}

[1.] Steven H. Strogatz, Nonlinear Dynamics and chaos : With applications to Physics, Biology, Chemistry and Engineering .

[2.] Michel Vellekoop ; Raoul Berglund, On intervals, Transitivity = Chaos, American Mathematical Monthly, Volume 101, Issue

[3.] Devaney R. L., An introduction to Chaotic Dynamical Systems, Second Edition, Addition Wesley, Redwood city, 1989

[4.] Kathleen T. Alligood, Tim D. Sauer, James A. Yorke, CHAOS - An introduction to dynamical systems, Springer Verlag, New Y

[5.] Saber N. Elaydi, Discrete Chaos, CHAPMAN \& HALL/CRC, 2000

[6.] Michael Brin \& Garrett Stuck, Introduction to dynamical systems, Cambridge University Press, 2002

[7.] Robert Gilmore \& Marc Lefranc, The topology of Chaos, John Wiley \& Sons. Inc. New York, 2002

[8.] Tien-Yien Li and James A. Yorke, 'Period three implies Chaos' American Mathematical Monthly, 1975.

[9.] E. J. Barbeau, Polynomials, Page 164

[10.] www.mathworld.wolfram.com 